\title{
Study on the Portable Oil-Water Interface Detector
}

\author{
Zejie WANG ${ }^{1, a}$, Jin $\mathrm{CHEN}^{2,}$,, Xuelei WANG ${ }^{3, c}, \mathrm{Ya} \mathrm{XU}^{4, \mathrm{~d}}$, Qiao $\mathrm{LI}^{5, \mathrm{e}}$ \\ ${ }^{1234}$ School of Mechanical Engineering, Jiangsu University, Zhenjiang, 212013, China \\ ${ }^{5}$ Jiangsu Yuanwang Instruments CO. LTD, Taizhou,225300, China. \\ aemail:512445649@qq.com, bemail:Chenjinjd@126.com, 'email: 614979024@qq.com, \\ demail: 912916874@qq.com, email: qtliqiao@126.com.
}

Keywords: oil-water interface; detector ; near-infrared spectrum; oil concentration

\begin{abstract}
In order to detect the accurate oil-water interface in the oil storage tank in real time, the paper designed a portable oil-water interface detector. It adopts the oil-water detection method based on the near-infrared absorption spectrum theory to determine the exact oil-water interface cut-off point through analyzing the changes of oil concentration in the emulsion between oil and water layers, thus determine the position of oil ,water and emulsified oil, improving the detection accuracy of the oil-water interface. And it provides a reliable basis for the water automatic discharge in oil separation tank and the management for the oil preservation and transportation system.
\end{abstract}

\section{Introduction}

In the production process of crude oil, oil will always contain certain water, it need to be sent to the crude oil storage tank to implement oil/water separation [1][2]. Due to the different proportion of oil and water, water in the crude oil will settle at the bottom of the tank and the oil will float on the upper, from the roof to the tank bottom, followed by air, oil, oil/water emulsion, water, sludge layer, the position of oil-water emulsion band is to change with the injection and discharge of the oil [3]. From the perspective of oil concentration, the different location of the emulsion, the oil concentration is different, the more close to the oil layer, oil concentration is higher, close to the water layer is on the contrary. According to different detection principles, the common oil-water interface detectors are:

(1) Differential pressure type interface instrument: through detecting the pressure in different positions of tank to reflect the oil-water mixture density in different positions. However, because of the effect of salinity, emulsifiers, and various kinds of polymer, the density of oil and water is very close (especially for heavy oil), and oil density is variable, this change is hard to obtain the real-time compensation in the instrument.

(2) Capacitive interface instrument: the main use of the principle of the change of the capacitor caused by dielectric change, the sensor is placed inside the tank to measure the different height positions of dielectric capacitors directly, capacitance and the interface height have a linear relationship, However, due to the problems of hanging oil, in particular heavy oil, the measuring error is large.

(3) RF admittance interface instrument: based on the theory of radio frequency impedance, through presenting impedance characteristics of the measured medium to determine the location of the oil-water interface. It has large measuring range and overcomes the adverse effects of salinity and hanging oil, but only through a conductivity parameter is difficult to reflect the state of oil-water emulsion completely.

The above oil-water interface detectors, their volume is big and not easy to carry to the field to conduct real-time detection, and detecting accuracy is not very good to meet filed requirements. To cure the above problems, this paper designs a portable oil-water interface detector. Using near infrared oil-water detection method, by analyzing the change of oil concentration between water and oil, to determine the oil-water cut-off point effectively and reduce the influence of emulsion 
belt. And it has the advantages of small volume, convenient carrying, simple detecting process, reducing the personnel labor intensity and high detection precision.

\section{The overall design of the portable digital oil-water interface detector}

In this paper, the design of oil-water interface detector is mainly composed of two parts shown in Figure 1, one part is detecting probe, probe has built-in ultrasonic detection circuit, using the $1 \mathrm{MHZ}$ piezoelectric ceramic ultrasonic transducers, according to the principle of that high frequency ultrasound in the air damping larger to distinguish air and liquid (oil and water) effectively; Near infrared detection circuit, by detecting the oil concentration changes between water and oil layers to determine the oil-water cut-off point, and then determine the position of the oil-water interface; the temperature detection circuit, detecting the temperature of medium that probe contact with. The other part is a hand-held terminal, it has built-in MCU2, and designed a display module, a magnetic rotary coding module, alarm module, and buttons. Magnetic rotary coding module determines the position of probe decreased according to the measurement of rotation angle ; The alarm module is mainly used to provide alarm sounds of different rhythm when detected different media, in order to prompt testing personnel to contrast the display results; The button is used to confirm the result of the detection and calibrate temperature; The display module can display the measurement results synchronously, including the properties of the medium air, oil, or water, the temperature of the medium and the position of the detecting probe. Detecting probe and hand-held terminal are connected by a special measuring scale with a metal wire built-in probe, the metal wire is used for signal transmission between two parts and the power supply of the detection probe.

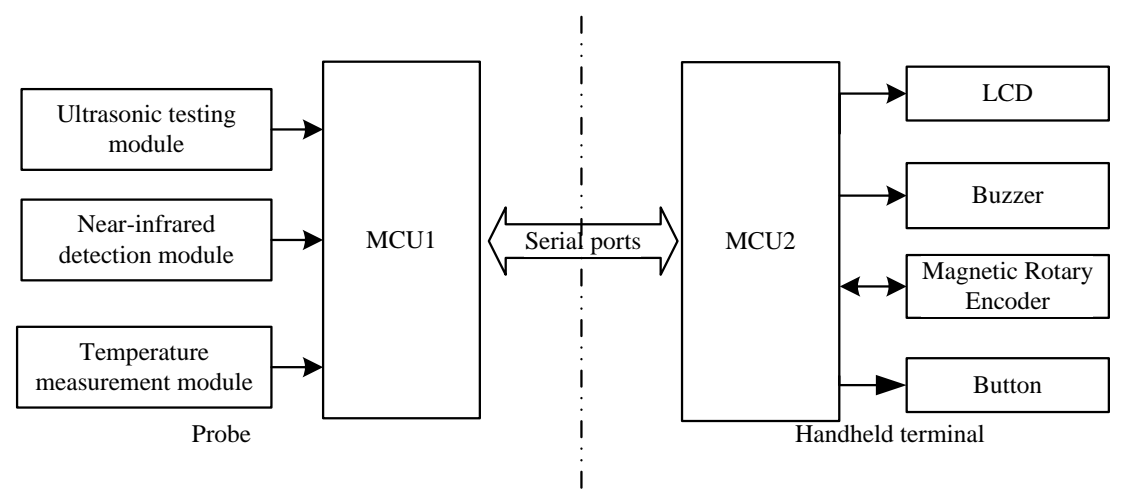

Fig.1. The overall design diagram

\section{Oil-water interface detection system}

The oil-water interface detection system is shown in Figure 2, when the oil tank internal completely static, air, oil and water layers are arranged sequentially from top to bottom of storage tank internal, and the emulsion layer exist between oil and water zones, under the control of hand-held terminal, the detecting probe of portable oil-water interface detector through the tank body from top to bottom and detect properties and temperature of the contacted medium in real time, when the detecting probe from the air into the oil layer, the probe will send specified electrical signal to the hand-held terminal through the serial port, a hand-held terminal recording liquid level height (h1), h1 is the oil level height. When the detection probe reach the water layer, the hand-held terminal will receive the corresponding medium change signal from the probe and record height (h2) at this time, h2 is the water level height, when the probe to the tank bottom, record height (h3) at this time. Generally, the height of tank is known, so can get h3 directly. Through the calculation, $\mathrm{h} 2-\mathrm{h} 1$ is the thickness of the oil layer, h3-h2 is the thickness of the water layer. h1 and h2 are measured by the hand-held terminal built-in magnetic rotary encoder. 


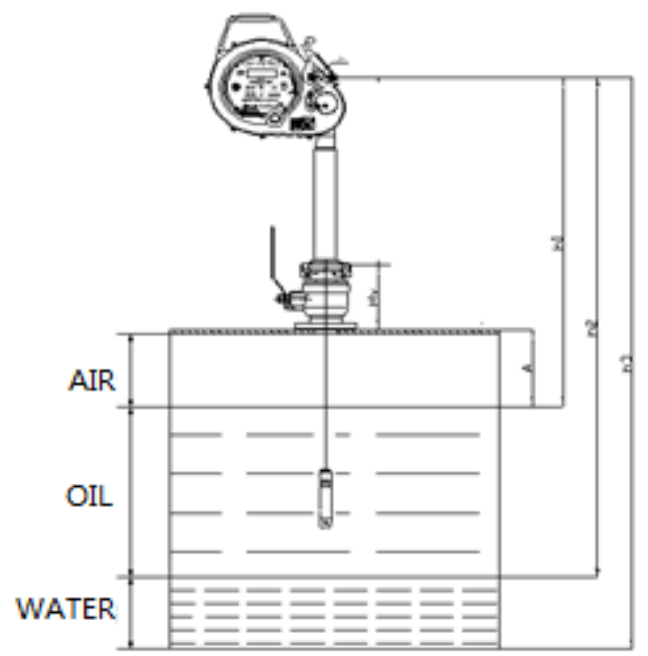

Fig.2. oil-water interface detection system

\section{Near infrared oil-water interface detection method}

Lambert-Beer's law is the basic law of light absorption, also be the basis of near infrared spectral analysis. A beam of monochromatic light irradiation on an absorbing medium surface and through a certain thickness of the medium, due to the medium absorbs a part of the light, the intensity of transmission light will be weakened.The greater concentration and the thicker of absorbing medium, the less intensity of the light. And the relationship of the formula:

$$
A=\lg \frac{I_{0}}{I_{t}}=\lg \frac{1}{T}=K \cdot \mathrm{L} \cdot \mathrm{C}
$$

A: absorbance I0:intensity incident light, It: intensity of transmitted light T: Transmittance K: absorption coefficient $(\mathrm{L} /(\mathrm{g} \cdot \mathrm{cm})) \mathrm{L}$ : thickness of the absorbing medium $(\mathrm{cm}) \mathrm{C}$ : concentration of light-absorbing substance $(\mathrm{g} / \mathrm{L})$

This paper used dual wavelength spectrophotometry based on the Lambert - Beer to detect oil and water medium [4]. Because in the oil-water detection, there may be some impurities, such as tiny particulates, that can block the optical channel and increase the random error of detection, causing a great influence on the measurement results. The dual wavelength spectrophotometric detection method can effectively reduce the interference of impurities. Selecting two appropriate wavelength of light, one as the determination wavelength, another as the reference wavelength, The impurities for the two detection wavelength absorbance are the same, the wavelength of determination is denoted $\lambda_{1}$, the reference wavelength is $\lambda_{2}$, Lambert - Beer law shows that when measuring wavelength is $\lambda 1$, the absorbance A1 that through some solution is:

$$
\mathrm{A} 1=\lg \frac{I_{\lambda 10}}{I_{\lambda 1 t}}=K_{11} b c_{x}+K_{12} b c_{y}\left(\mathrm{~A}_{1 \mathrm{y}}\right)
$$

Reference wavelength $\lambda 2$ through certain solution's absorbance A2:

$$
\mathrm{A} 2=\lg \frac{I_{\lambda 20}}{I_{\lambda 2 t}}=K_{21} b c_{x}+K_{22} b c_{y}\left(\mathrm{~A}_{2 \mathrm{y}}\right)
$$

In the formula (2) and (3), $I_{\lambda 10}$ and $I_{\lambda 1 \mathrm{t}}$ are the incident light intensity and the transmitted light intensity of the wavelength $\lambda 1, I_{\lambda 20}$ and $I_{\lambda 2 \mathrm{t}}$ are the incident light intensity and the transmitted light intensity of the wavelength $\lambda 2, \mathrm{~K}_{11} 、 \mathrm{~K}_{12} 、 \mathrm{~K}_{21}$ and $\mathrm{K}_{22}$ represent absorbance coefficient under different wavelengths, $A_{1 y}$ and $A_{2 y}$ stand for the influence of impurities on the absorbance, $c_{x}$ is the concentration of the tested substance, $c_{y}$ is the concentration of the impurities. When selecting the appropriate wavelength make $A_{1 y}$ equal to $A_{2 y}$, formula (2)and (3) make subtraction .

$$
\Delta A=A 1-A 2=K_{11} b c_{x}-K_{21} b c_{x}=\left(K_{11}-\mathrm{K}_{21}\right) \mathrm{b} c_{x}
$$


Formula (4) shows that absorbance only have a linear relationship with the tested concentration, eliminating or reducing the interference of impurity. According to the different spectral characteristics of oil and water in the near infrared region, In this article uses a 910nm near-infrared light as the measurement wavelength, a $650 \mathrm{~nm}$ red light as reference wavelength.

When the dielectric thickness and the absorption coefficient of light absorption is determined, the absorbance of the medium (A) is directly proportional to the concentration of the medium(c), that means by detecting the absorbency to calculate the medium concentration of oil, then according to the concentration values and the concentration change of oil to find appropriate oil-water cut-off point, to reduce the impact of emulsified, and to determine the accurate location of the oil-water interface.

In spectrum analysis, in order to get the accurate quantitative relationship, need to establish the mathematical model, and analyzes the reliability of the model. Considering the absorbance and concentration of oil have a linear relationship, as well as test data collected including information on two wavelengths, and the impact of impurities to the experimental, the mathematical method adopted is partial the method of least squares. The partial least square method is the linear regression model established between the dependent variables principal and independent variable principal component, indirectly reflecting the relationship between variables and independent variables [5]. In the quantitative analysis of oil concentration and absorbance, making the spectrum matrix $\mathrm{X}$ and the concentration matrix $\mathrm{Y}$ decomposition, the model:

$$
\left.\begin{array}{l}
X=T P+E \\
\mathrm{Y}=\mathrm{UQ}+\mathrm{F}
\end{array}\right\}
$$

$\mathrm{T}$ and $\mathrm{U}$ are $\mathrm{X}$ and $\mathrm{Y}$ matrix's scoring matrix; $\mathrm{P}$ and $\mathrm{Q}$, respectively, $\mathrm{X}$ and $\mathrm{Y}$ matrix's loading matrix; $\mathrm{E}$ and $\mathrm{F}$ are the $\mathrm{X}$ and Y matrix's fit residuals matrix PLS, the second step of PLS is making $\mathrm{T}$ and $\mathrm{U}$ to the linear regression:

$$
\left.\begin{array}{l}
\mathrm{U}=\mathrm{TB} \\
\mathrm{B}=\left(\mathrm{T}^{\mathrm{T}}\right)^{-1} \mathrm{~T}^{\mathrm{T}} \mathrm{Y}
\end{array}\right\}
$$

In the forecast, according to the $\mathrm{P}$ to achieve the scoring matrix of unknown sample spectrum matrix, according to the following type to get the predictive value of concentration:

$$
\mathrm{Y}_{\text {UNknown }}=\mathrm{T}_{\text {UNknown }} \mathrm{BQ}
$$

In the data processing, the establishment of PLS mathematical model is based on the NIRSA software developed by Jiangsu University, using a total of 70 samples, of which 70 per cent of samples (49) as a calibration sample set, and the remaining 21 as a predictor sample collection, the predicted value and the real value relationship shown in Figure 3, the correlation coefficient: 0.998, the linear prediction equation: $\mathrm{Y}=0.9961 \mathrm{X}-1.118$, RMSEP: 13.1026, prediction error check value $\mathrm{T}: 1.5423$, T-sided test critical value: $\mathrm{T}(20,0.05)=2.0860$

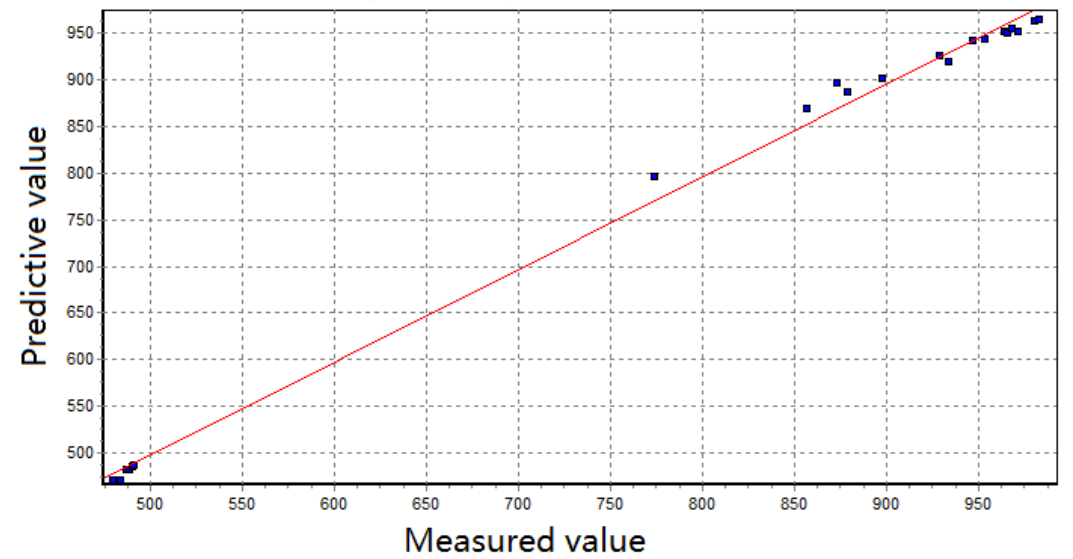

Fig.3 .model predicted results

Through the analysis of the above results, the oil concentration model based on partial least square method is effective and can predict the oil concentration according to the model, to get a quantitative relationship between oil concentration and the absorbance. Through the quantitative 
analysis of concentration combined with the agreement of water layer and pure oil can determine an appropriate interface boundary to reduce the effect of emulsification belt and improve the accuracy of oil water interface.

\section{Design of Software}

The software design of the oil-water interface detector mainly includes signal acquisition, including the infrared signal acquisition, signal acquisition and temperature signal acquisition, data processing, the serial communication, display and alarm of detect results etc. The overall flow chart of the system is shown in Figure 4.

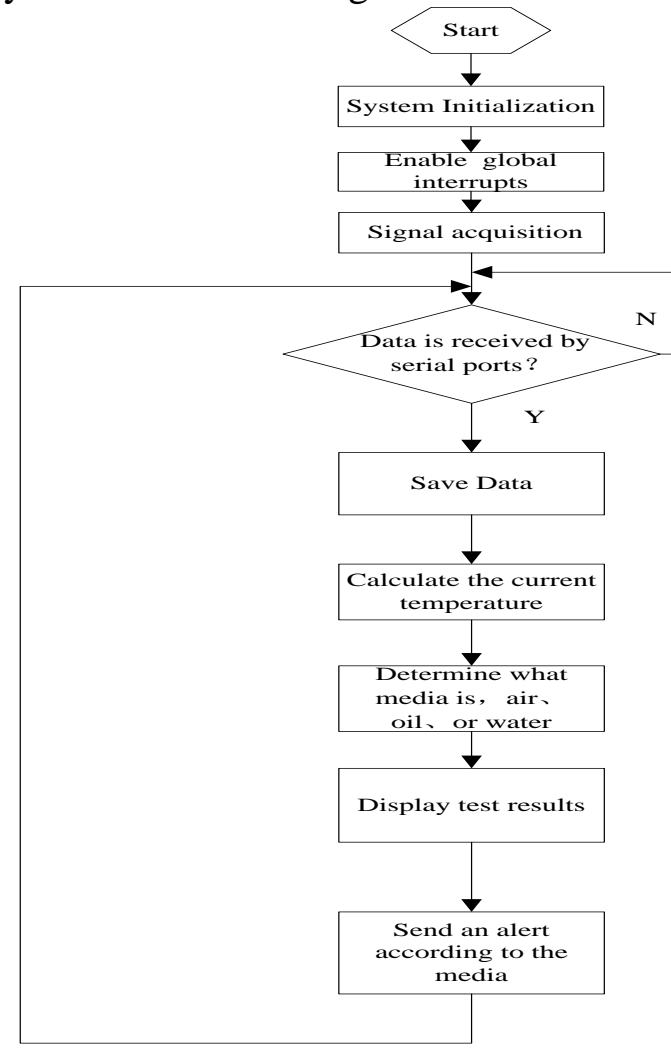

Fig.4. The overall flow chart of the system

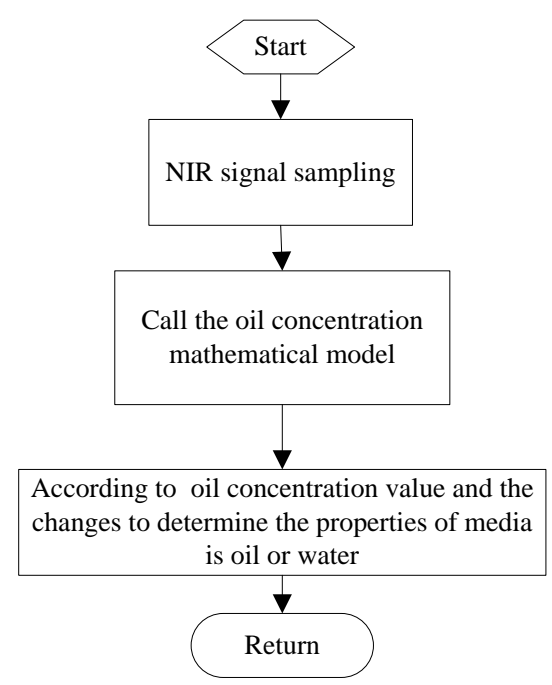

Fig.5. The flow chart near-infrared detection

The near infrared oil-water detect software design is shown in Figure 5, the collected near infrared signal converts into the corresponding oil concentration by calling the oil concentration mathematical model, determine the media is water or oil according to the concentration value and change, then determine the position of oil-water interface.

\section{Field testing of portable water interface detector}

According to figure 2 to establish oil-water interface detection system, according to Table 1 to mix into 8 kinds of test sample solution, each test sample liquid in the container by the oil and water mixing ratio of 1:8, each test subject only allow to do after oil-water interface post clear completely. The actual position of oil-water interface was measured with a standard length tape, and the detection probe with no more than $10 \mathrm{~mm}$ / s speed moves down, when it reaches oil-water interface, stop moving and press the stop-watch to start timing. When hearing the buzzer change the sound , pressing the stop-watch to stop the clock immediately, and record the location of interface. The readings and reaction time are recorded in Table 1 below. 
Tab.1. The testing results of Portable oil-water interface detector

\begin{tabular}{|c|c|c|c|c|c|c|c|c|}
\hline \multirow[t]{3}{*}{ Water } & \multicolumn{4}{|c|}{$\begin{array}{l}\text { Fresh water } \\
\left(\rho=1.00 \mathrm{~g} / \mathrm{cm}^{3}\right)\end{array}$} & \multicolumn{4}{|c|}{$\begin{array}{c}\text { Salt water } \\
\left(\rho=1.025 \mathrm{~g} / \mathrm{cm}^{3}\right)\end{array}$} \\
\hline & \multicolumn{3}{|c|}{ Accuracy $(\mathrm{mm})$} & \multirow{2}{*}{$\begin{array}{l}\text { reaction } \\
\text { time (s) }\end{array}$} & \multicolumn{3}{|c|}{ Accuracy ( mm) } & \multirow{2}{*}{$\begin{array}{l}\text { reaction } \\
\text { time }(\mathrm{s})\end{array}$} \\
\hline & $\begin{array}{l}\text { Actual } \\
\text { value }\end{array}$ & $\begin{array}{l}\text { Measure } \\
\text { value }\end{array}$ & error & & $\begin{array}{l}\text { Actual } \\
\text { value }\end{array}$ & $\begin{array}{l}\text { Measure } \\
\text { value }\end{array}$ & error & \\
\hline No.93diesel & 399 & 396 & -3 & 1 & 401 & 402 & +1 & 1 \\
\hline No.0 diesel & 399 & 401 & +2 & 1 & 402 & 400 & -2 & 1 \\
\hline No.2crude & 400 & 392 & -8 & 1.5 & 402 & 395 & -7 & 2 \\
\hline 180CST & 400 & 394 & -6 & 2 & 403 & 398 & -5 & 2 \\
\hline
\end{tabular}

From the table1, for No. 93 and No. 0 diesel oil with relatively low concentration, it has a high accuracy of oil-water interface detection, with the error $\pm 3 \mathrm{~mm}$ and response time $\leqslant 1 \mathrm{~s}$, the concentrations of No.2 crude and 180CST are relatively high, the measurement error is about \pm $6 \mathrm{~mm}$, response time $\leqslant 2 \mathrm{~s}$, and measurement values in fresh water and salt water are almost equal, difference is about $1 \mathrm{~mm}$.Compared with national standard that the measurement accuracy of oil-water interface detector within $25 \mathrm{~mm}$, reaction time is not more than $3 \mathrm{~s}$ and the traditional oil-water interface detectors' precision (about $\pm 10 \mathrm{~mm}$ ), the portable oil-water interface detector in this paper has higher detection precision and fast responding speed.

\section{Conclusion}

This paper designed a portable detector for oil-water interface, adopting near infrared oil-water detection method, and established the quantitative relationship between the absorbance and oil concentration, according to the oil concentration in the process of detection to determine the accurate oil-water interface position. Compared with common oil-water interface detectors, it can reduce the impact of oil-water emulsion layer band and improve the detection accuracy, it also has the advantages of simple structure, small volume, easy to carry, and is suitable for the real-time field oil-water interface detection.

\section{References}

[1]Chen Hui-li. Oil-water interface detector research and design[D], Wuhan, Wuhan University of Technology, 2013.

[2] Zhou Zhao-yan. Research on oil-water interface online detection system[D], Shenyang: Northeastern University, 2011.

[3] REN Lei, Chen Xiang-guang, Liu chun-tao. Research and Application on the measurement method of crude storage tank oil-water interface[J]. Instrument Technology, 2008,29 (10): 2086-2091.

[4] Xu Jun, The design and research of spectrophotometric absorbance detection device based on dual-wavelength [D], Hangzhou; Zhejiang University of Technology, 2013.

[5] Su Yue, Guo Yin-long, a new method to determine the principal component scores in partial least squares method[J], Computers and Applied Chemistry, 2001,18 (3), 237-240. 